\title{
ARTICLE
}

\section{In-patient services for people with intellectual disability and mental health or behavioural difficulties}

\author{
John Devapriam, Alan Rosenbach \& Regi Alexander
}

\begin{abstract}
John Devapriam is a consultant psychiatrist in intellectual disability with Leicestershire Partnership NHS Trust and National Professional Advisor at the Care Quality Commission (COC). Alan Rosenbach is the Senior Strategy Lead at the COC. Regi Alexander is a consultant psychiatrist in intellectual disability at Partnerships in Care's learning disability services, Diss, Norfolk and an Honorary

Senior Lecturer at the University of East Anglia. Correspondence $\mathrm{Dr}$ John Devapriam, Leicester Frith Hospital, Mansion House, Groby Road, Leicester LE3 90F, UK. Email: john.devapriam@leicspart.nhs.uk
\end{abstract}

\begin{abstract}
SUMMARY
Over the past few decades, care for people with intellectual disability in the UK has moved from long-stay hospitals to the community. As in the general population, a number of these people have mental health and behavioural difficulties for which they may require in-patient services. Consequently, psychiatrists need to be aware of the in-patient mental healthcare provision for these individuals. This article describes the different categories of in-patient bed for patients with intellectual disability and ways to monitor the quality and outcomes of in-patient care.

\section{LEARNING OBJECTIVES}

- Understand the need for in-patient treatment of people with intellectual disability and mental health, behavioural or forensic problems

- Understand the different categories of in-patient services for people with intellectual disability

- Understand the ways of monitoring standards and measuring outcomes for these services and people

\section{DECLARATION OF INTEREST}

J.D. is the National Professional Advisor on Learning Disability to the Care Quality Commission (COC). A.R. is the Senior Strategy Lead at the CQC and has worked on the CQC's contribution to system change for intellectual disability services. R.A. was Chair/Editor and J.D. was co-editor of the report People with Learning Disability and Mental Health, Behavioural or Forensic Problems: The Role of In-Patient Services (Royal College of Psychiatrists 2013).
\end{abstract}

Historically, many people with intellectual disability were cared for in long-stay hospitals for those with a 'mental handicap'. All their physical and mental health needs were meant to be provided for in these institutions and they seldom came into contact with generic services. Following the deinstitutionalisation movement of the 1980s, these long-stay hospitals were closed. The number of long-stay beds fell from around
64000 in 1970, to well under 10000 by 2001 (NHS England 2013) and around 3954 by 20122013 (Royal College of Psychiatrists 2013). The role of psychiatrists involved in this field changed too. From being 'mental handicap doctors' who dealt with all aspects of individuals' physical and mental healthcare, they now became specialists primarily responsible for managing their mental health and behavioural problems.

Current government policy in the UK expects that, wherever possible, people with intellectual disability should be enabled to use generic health services (Department of Health 2001). Although the equity of access that mainstreaming brings is very pleasing, it is meaningless if not accompanied by equity of outcome. This is a particular problem for those with intellectual disability who have mental health and/or severe behavioural problems. Compared with the general population, people with intellectual disability have higher rates of mental health problems (Cooper 2007; Morgan 2008), their clinical presentations are often unique and their responses to treatment potentially different. In many cases, therefore, the identification and treatment of their comorbid mental health problems require specialist expertise both in generic and specialist settings (BjelogrlicLaakso 2014; Department of Health 2015). In this regard, the UK with its specialist postgraduate training in the psychiatry of intellectual disability is in a position of unique strength. It is because of an acknowledgement of these particular mental healthcare needs that this country has introduced specialised intellectual disability teams providing a range of services in both community and inpatient settings (Lindsey 2000).

In-patient units for people with intellectual disability and mental health or behavioural problems (described as assessment and treatment units) in the UK have come under scrutiny as a result of BBC television's Panorama programme 'Undercover care: the abuse exposed'. Following its broadcast in May 2011, a number of reports (Department of Health 2012a,b) described 
assessment and treatment units as a new form of institutional care that had no place in the 21st century and concluded that there were too many people staying for too long in these units. However, describing all in-patient services for people with intellectual disability as 'assessment and treatment units' ignores the existence of various categories of bed provision that serve completely different functions. This article seeks to clarify in-patient services for the treatment of mental health and behavioural problems in people with intellectual disability, focusing on why they are needed, the different bed categories and ways of monitoring standards and outcomes.

\section{The need for in-patient services}

The need for in-patient services to treat people with intellectual disability has to be considered in the context of the mental health difficulties and challenging or problem behaviours in this population.

\section{Comorbid mental health problems}

Intellectual disability is a condition characterised by significant impairments of both intellectual and adaptive functioning and an onset before the age of 18 (World Health Organization 1992). The term 'disorders of intellectual development' is also used and the UK health services currently use the term 'learning disability' to describe this group. About $1-2 \%$ of the general population will have an intellectual disability (Emerson 2010). The degree of intellectual disability is categorised as mild, moderate, severe or profound, with over $90 \%$ of those affected falling within the mild range (Department of Health 2001). In England, with a population of about 53 million, around 900000 adults have an intellectual disability, but only 191000 (21\%) of these are in contact with intellectual disability services (Emerson 2010) and $3035(0.3 \%)$ are receiving treatment in in-patient psychiatric units at any point in time (Health and Social Care Information Centre 2013) (Fig. 1).

People with intellectual disability have high rates of comorbid mental health problems. Epidemiological studies have suggested a prevalence rate of 31 to $41 \%$ (Cooper 2007; Morgan 2008). For those treated in non-secure hospital settings, figures from specialist intellectual disability in-patient units show rates of comorbid major mental illness ranging from 50 to $84 \%$ (Alexander 2001; Tajuddin 2004; Hall 2006a,b). This is in addition to other comorbid conditions, such as autism spectrum disorders, attentiondeficit hyperactivity disorder (ADHD), personality disorders and substance misuse. Similarly high figures are reported for those treated in secure hospital services for people with intellectual disability: up to $50 \%$ have a personality disorder, up to $30 \%$ have an autism spectrum disorder, about 30-50\% have a major mental illness, about 30-50\% have substance misuse/dependence and about 20\% have epilepsy (Alexander 2002, 2006; Plant 2011). Some of these conditions present with challenging behaviours and some do not.

\section{Challenging behaviour}

Challenging behaviour has been defined as behaviour that, because of its intensity, frequency or duration, poses a threat to the quality of life and/ or physical safety of the individual or others and is likely to lead to restrictive or aversive responses or exclusion (Royal College of Psychiatrists 2007). It is a socially constructed, descriptive concept that has no diagnostic significance and makes no inferences about aetiology. It encompasses heterogeneous behavioural phenomena in different groups of people and may be either unrelated to psychiatric disorder or a primary or secondary manifestation of it (Xenitidis 2001). In people with intellectual disability who come into contact with health services, it can range from stereotypies and self-injury in a person with a profound intellectual disability to violent offences in someone with a mild intellectual disability. Not all of those who present with challenging behaviour that involves aggression are arrested, charged or convicted (Royal College of Psychiatrists 2014).

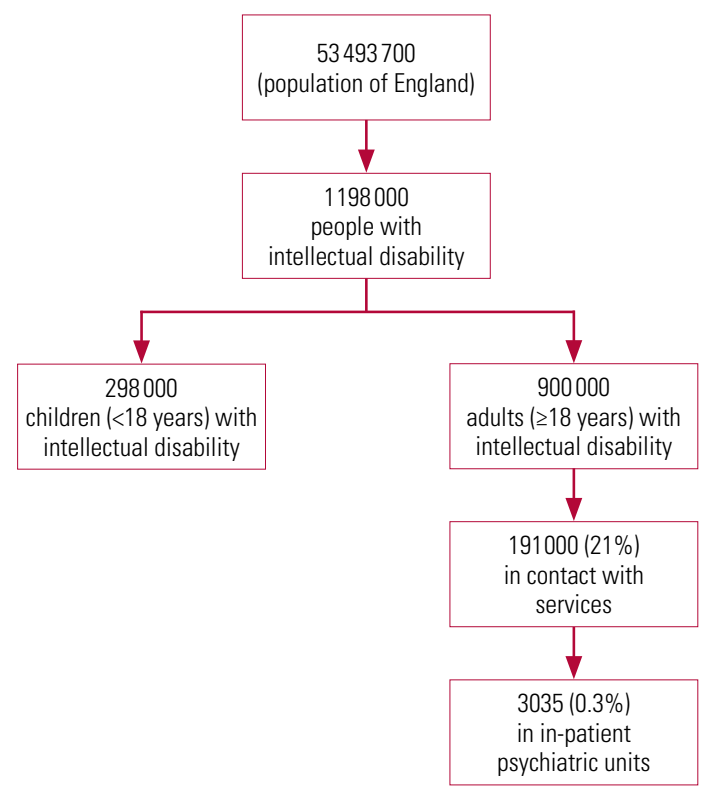

FIG 1 Community and in-patient service use by people with intellectual disability in England (Emerson 2010; Health and Social Care Information Centre 2013). 


\section{Addressing challenging behaviour}

The therapeutic approach to challenging behaviour has been well described and emphasises the use of the least restrictive community resource wherever possible (Royal College of Psychiatrists 2007). Inpatient admission to a psychiatric unit is required only if the risk posed by the behaviour is of such a degree that it cannot safely be managed in the community or if an early assessment is required in a safe setting where the patient can be monitored by specialists to ascertain the underlying cause of the behaviour, which may very well be a mental health problem.

The assumption that all behavioural problems in people with intellectual disability were a consequence of institutional lifestyles and that they would diminish once community care was introduced may be flawed (Holland 2002). People with intellectual disability and mental health or behavioural difficulties living in the community require access to in-patient services for a number of reasons. First, behaviours previously hidden or tolerated within institutions are more visible in the community and they are more likely to have adverse consequences (Moss 2002). An increased societal aversion to risk (Carroll 2004) makes this dynamic more potent. Challenging behaviour, whether it is aggression or self-injury, can pose a level of risk that is deemed unacceptable in a community setting. Consequently, in-patient settings of varying degrees of security may be needed for varying periods of time. The guiding principle is to choose the least restrictive option.

Second, any patient who is seen as 'liable to be detained' under the Mental Health Act 1983 will by law require a hospital bed $(R v$ Hallstrom ex parte $W$ [1986] 2 All ER 306).

Third, as already mentioned, people with intellectual disability have higher rates of comorbid psychiatric disorder. Their clinical presentations are often a complex mix of intellectual disability, mental illness, personality disorder, pervasive developmental disorder and other developmental disorders (e.g. hyperkinetic disorder). The natural course of mental disorders suggests that these individuals might experience periods of crisis as well as long-term behavioural symptoms that persist despite treatment. In both situations, they may need a safe setting where professionally qualified staff can treat them with continuous nursing observation, behavioural monitoring and multidisciplinary professional input beyond what can be reasonably achieved safely in the community.

Finally, many people with intellectual disability and mental health problems also have a wide range of physical disorders, including epilepsy (Emerson 2010), that further complicate their presentations. In some people who present with challenging behaviour, these mental and physical health problems are intricately linked and often it can be difficult to tease out whether the presentation is because of an underlying organic (physical) condition. In many of these complex presentations, an accurate diagnosis and effective treatment require continuous nursing observation, physical investigations, and medical and psychiatric expertise in an in-patient setting.

\section{Types of in-patient bed}

A report published by the Faculty of Psychiatry of Intellectual Disability at the Royal College of Psychiatrists (2013) gives a detailed description, illustrated with a number of case vignettes, of the purpose and functions of the different types of in-patient bed for people with intellectual disability in the UK.

These different categories of hospital bed (Box 1) are best understood within the context of a tiered-care model of service provision, with tiers 1 (liaison working with other agencies) to 3 (intensive case management in the community) constituting community intellectual disability services and tier 4 constituting the in-patient element of care (Royal College of Psychiatrists 2011) (Fig. 2). For a smooth patient journey through these tiers, it is generally acknowledged that health and social care commissioners and providers must take a 'whole-systems' approach to commissioning and providing care.

Until recently category-specific information on bed provision for people with intellectual

BOX 1 Bed categories in the UK

In-patient beds for people with intellectual disability and mental health and/or severe behavioural problems are divided into 6 categories

Category 1: High, medium and low secure forensic beds

Category 2: Acute admission beds in specialised intellectual disability units

Category 3: Acute admission beds in generic mental health settings

Category 4: Forensic rehabilitation beds

Category 5: Complex continuing care and rehabilitation beds

Category 6: Other beds, including those for specialist neuropsychiatric conditions and short breaks

(Royal College of Psychiatrists 2013) 
disability has been limited. This is because the information that healthcare providers must supply when they seek registration with the Care Quality Commission (CQC) to operate in the UK has been shaped by the Health and Social Care Act 2008. Consequently, they need give only limited details about the categories and purpose of bed provision for people with intellectual disability and mental health or behavioural problems. It is now acknowledged that the Royal College of Psychiatrists' categorisation of bed types as described in Box 1 is a cogent articulation of the range of in-patient environments serving different assessment and treatment purposes. As an endorsement of this, the recent Learning Disability Census (Health and Social Care Information Centre 2013) collected data from providers using these categories.

\section{Category 1 (high, medium and low secure beds)}

Secure beds are for patients who pose a level of risk assessed as requiring the physical, relational and procedural security of a high, medium or low secure unit. The general characteristics of these units are described elsewhere (Kennedy 2002; Phillips 2007; Tucker 2007; Royal College of Psychiatrists 2013). All patients accessing these beds tend to be detained under the Mental Health Act 1983 (amended 2007). These services offer an integrated, multidisciplinary model of support within an environment that emphasises care and treatment rather than punishment (Hollins 2000).

The decision whether or not a person needs a secure bed often depends on clinical judgements about risk and the attitudes of professionals working in the criminal justice, health and social care systems. These attitudes and decisions are in turn shaped by the availability of resources and hence if less restrictive in-patient settings are unavailable or inaccessible, then the use of secure beds can go up.

The length of stay in secure units is dictated by several factors, including public attitudes to risk, duration of treatment programmes, response to treatment and the availability of appropriate stepdown facilities. A streamlined process exists in some regions for access to these units: a clinician (usually from the patient's local area) carries out an access assessment and the patient's relational, structural and procedural security needs and treatment requirements are established before a commissioning decision is made. In addition to reviews by first-tier tribunals (mental health), these placements are also reviewed at regular intervals by the commissioners who source and fund the placement and the access assessors to see whether patients can move to less restrictive placements.

\section{Category 2 lacute admission beds in specialist intellectual disability units) and category 3 (acute admission beds in acute mental health wards or in such wards with a specialist intellectual disability function)}

Category 2 and category 3 beds are intended for the assessment and treatment of acute severe mental health and/or behavioural problems which pose a risk that cannot be safely managed in the community, but does not meet the threshold to be considered for a secure bed. These units complement community services and are to be used for a short period to mitigate risk therapeutically and to enable a thorough assessment of mental health needs by specialist staff in a controlled environment. Access to either of these bed categories should be informed by clinical need and patient choice.

The literature comparing these two models is well summarised in two elegant structured reviews (Chaplin 2004, 2009). The studies included were controlled trials or descriptive surveys drawn from the UK, USA, Canada and Australia. The main conclusions are summarised in Box 2.

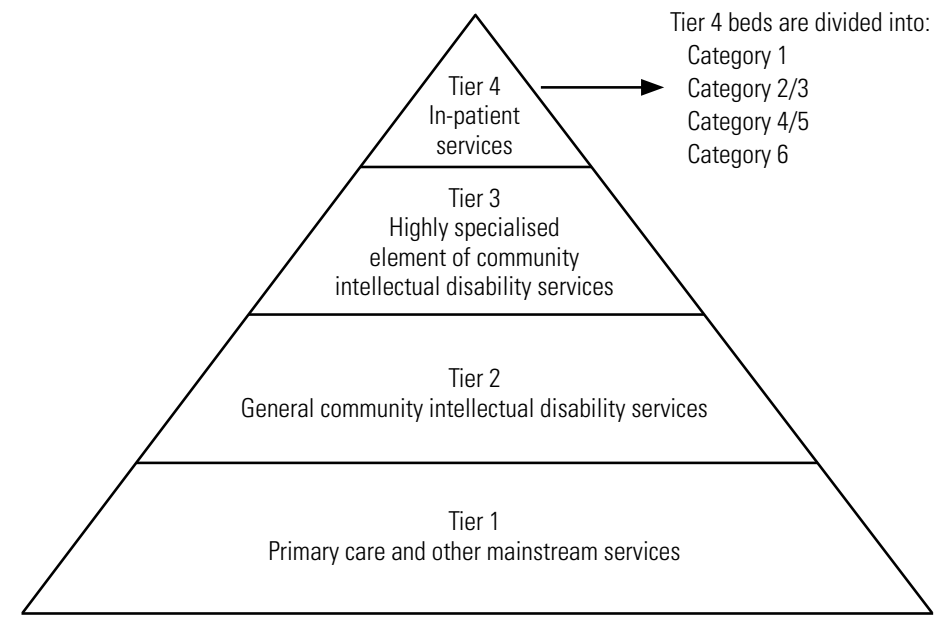

Tier 1: primary care and other mainstream services. It is the tier of service provision that serves the general health, social care and educational needs of people with intellectual disability and their families. The community intellectual disability team and the psychiatrist have limited direct clinical contact with patients in this tier. Nevertheless, they are involved in activities that may influence patients' care and interacting with this tier is essential to the training of intellectual disability psychiatrists.

Tier 2: general community intellectual disability services. At this level, individuals with intellectual disability start to use specialist intellectual disability services. Most specialist services are provided jointly by health and social services or are moving towards such a model.

Tier 3: a highly specialised element of community intellectual disability services. It includes areas of specialised needs such as epilepsy, dementia, challenging behaviour, pervasive developmental disorders and out-patient forensic services.

Tier 4: specialist in-patient services. It includes all specialist in-patient services for people with intellectual disability, ranging from local assessment and treatment services to high secure forensic services.

Tiered model of care for intellectual (learning) disability services (Royal College of Psychiatrists 2011: p. 23) 
BOX 2 Category 2 and 3 beds - the evidence

- There is no conclusive evidence to favour either model (i.e. category 2 or category 3 beds)

- The two models serve different types of patient and this partially explains the differences in length of stay

- People with severe intellectual disability were not well served by generic services (i.e. category 3 beds)

- There was a worse outcome for people with intellectual disability in generic settings, particularly in the older studies; this poor outcome was improved if a specialist intellectual disability component was introduced into the generic setting

- Generic psychiatric care for people with intellectual disability is unpopular, especially with carers; again, this is improved by specialist input

- The provision of generic psychiatric care without specialist intellectual disability input is not sufficient to meet the needs of people with intellectual disability

(Chaplin 2004, 2009)

\section{Category 4 (forensic rehabilitation) and category 5 (complex continuing care and rehabilitation)}

These two categories refer to in-patient provision for patients whose mental health problems and behavioural difficulties remain intractable despite evidence-based treatment. These patients continue to need the structure, security and care offered by a hospital setting for long periods of time. Category 4 is mostly for people who have stepped down from category 1 beds, but who show enduring risky behaviour towards themselves or others. Many of these patients have committed serious offences and may be subject to restrictions from the Ministry of Justice. Although they have gone through offence-specific and other treatment programmes, their current risk assessments still emphasise the need for robust external supervision and ongoing treatment. They tend to have long durations of stay, often running into years, but it is the availability of category 4 beds, often in locked or open community units, that allows them to receive treatment in a less restrictive setting than a secure unit.

Category 5 is mostly for people who have undergone the initial intensive treatment process. Their diagnostic and psychological formulations are available and they have received a range of biopsychosocial treatments. However, for a variety of reasons (including treatment-refractory enduring mental illness, severe behavioural challenges that have not responded adequately to treatment, ongoing risks of neglect or vulnerability, and persisting risks to the safety of others), a safe transition into the community has not been possible even with adequately resourced community provisions. The provision of a stable, structured and predictable environment with qualified staff who can continue to offer physical and psychosocial treatments that incorporate positive risk-taking offers the best quality of life to these patients. Category 5 thus provides a process of rehabilitation and re-skilling for a transition to community settings. This is usually tailored to each individual's pace of progress, so durations of stay tend to be long. If these beds were not available, the potential consequence might be 'revolving door' patterns of hospital admission.

Category 4 and 5 beds are not unique to those with intellectual disability. Their definitions closely mirror those of the longer-term complex/ continuing care units described by the NHS Confederation (2012) in its report Defining Mental Health Services.

\section{Category 6 (other types of bed)}

This category includes specialist beds for neuropsychiatric conditions such as epilepsy and movement disorders. At present, this service provision is limited to a few specialised national units.

\section{Current intellectual disability in-patient provision in England}

In 2013 there were 3901 intellectual disability beds across four NHS regions in England: 2369 category 1; 814 category 2; 593 categories 4/5; and 125 category 6 (Royal College of Psychiatrists 2013). These include all NHS and independent sector provision for forensic and non-forensic services and represent an almost $90 \%$ reduction from a high of over 33000 beds in the NHS sector alone in 1987-1988 (NHS England 2013). The occupancy rate for these beds, although difficult to determine precisely, is estimated to be around $80 \%$, which is in keeping with the best practice guidelines for bed occupancy in in-patient mental health settings (Royal College of Psychiatrists 2011).

The Learning Disability Census (Health and Social Care Information Centre 2013) reported that there were 3250 people with intellectual disability in in-patient units across 104 service locations in England on 30 September 2013. The level of response and numbers were consistent with the last Count Me In census in 2010 (Care Quality Commission 2011). A time series analysis in the latter covering the years 2006-2010 showed that the number of people with intellectual disability and comorbid autism in in-patient units on the census days fell from 4435 in 2006 to 3376 in 2010. Although the trend shows a 
downward trajectory there are still too many patients who spend long periods in in-patient units, some of which are considerable distance from their home communities: 60\% of these patients (1949) had been in hospital for a year or more and $18 \%$ (570) were in hospitals $100 \mathrm{~km}$ or more from their residential postcode. Further analysis of length of stay for each bed category in these units is required. This is because a length of stay appropriate for a category 1 forensic bed may be thoroughly inappropriate for a category 2 or 3 acute admission bed. A notable change from the 2010 Count Me In census is that in 2013 the majority of individuals (76\%, 2481 people) were in-patients on wards that were predominantly for people with intellectual disability.

Although economies of scale may hamper efforts to provide all categories of bed in every district, the emphasis should be on the provision of in-patient services as close to the person's place of residence as possible. When planning service provision, it is therefore important to consider all in-patient beds, whether 'secure' or 'non-secure', as a whole to ensure smooth transition of care between community and in-patient services depending on patients' needs. It is good practice to commission and provide category 2, 3, 4 and 5 beds locally to enable the involvement of local teams and communities, and category 1 beds regionally. It has been suggested that, in addition to welldeveloped community teams, 6-7 in-patient beds across all six categories may be needed per 100000 population, although the number will differ greatly depending on local service configurations (Royal College of Psychiatrists 2013). If, in the absence of significantly improved community services, the less restrictive in-patient facilities (categories 2-5) are cut down, then many more people will potentially have unmet needs that compromise their mental health and safety and could end up in far more restrictive secure beds (category 1). This may be one of the reasons for the higher numbers in category 1 compared with categories $2-6$ in the recent census (Health and Social Care Information Centre 2013).

At present, 70\% of secure (category 1) beds are in low secure units. Since the provision of relational and procedural security is often more important than physical security for people with intellectual disability, many patients currently in these beds could move to the less restrictive rehabilitation beds (category 4 or 5). At present, this is problematic because the commissioning streams are different and there is significant geographical variation in the current distribution of beds.
A number of patients in category 4 and 5 beds (forensic rehabilitation and complex continuing care) stay in hospital for very long periods because, apart from therapeutic care, they also need continuous supervision for the protection of the public. If this type of supervision were legally enforceable in the community, without it amounting to the legal standard for de facto detention or deprivation of liberty, then they could very well live in the community.

\section{Monitoring standards and outcomes}

Assessing the quality of service provided in inpatient units rests on two key questions - first, is the treatment carried out to an adequate standard as defined by current clinical practice, and second, does the treatment work.

Accreditation tools focus on process variables in in-patient units and ensure that clinical practice is in keeping with standards approved by peers. There are several such tools, including the Royal College of Psychiatrists' Accreditation for Inpatient Mental Health Services in Learning Disability (AIMS-LD) for categories 2, 3 and 5 (Cresswell $2010)$ and the peer review accreditation process for categories 1 and 4 (Phillips 2007; Tucker 2007, 2012).

The measurement of process variables has to be supplemented with information about whether treatment provided in these settings works. A minimum data-set of outcome variables that cover measures of clinical effectiveness, patient safety and patient experience has been determined for this purpose (Royal College of Psychiatrists 2013: Tables 3 and 4, free online access).

It is important that these in patient services have robust arrangements for clinical governance to ensure that they are safe, effective and provide a positive patient experience. A number of good practice guidelines are listed in Box 3.

\section{Conclusions}

Currently in the UK, the vast majority of people with intellectual disability live fairly independent lives in the community, with only about $21 \%$ of adults with the condition having any contact with specialist intellectual disability services. However, they do have high rates of comorbid mental health problems: epidemiological studies show a prevalence rate of 31-41\% (Cooper 2007; Morgan 2008).

With well-resourced and well-trained community teams, these problems can be adequately treated in the community setting and unnecessary psychiatric hospital admissions can be avoided. Even with very good community teams, however, 


MCO answers
$1 \mathrm{c} \quad 2 \mathrm{~b} \quad 3 \mathrm{c} \quad 4 \mathrm{a} \quad 5 \mathrm{c}$

\section{B0X 3 Good practice points}

1 Service providers, commissioners and policy makers should be aware of the different categories of bed: these serve very different functions and any accreditation or inspection reports by regulatory authorities should specify bed categories.

2 A choice of both generic and specialist intellectual disability beds should be available for people with intellectual disability and mental health or behavioural problems who require acute in-patient psychiatric treatment. This choice should be driven by individuals' health and social care needs.

3 Regional commissioning strategies should focus on care pathways that include both well-developed community services and all six categories of in-patient bed.

4 To reduce the need for admission and shorten the length of stay in hospital, intellectual disability healthcare providers, mental health providers and local authorities should adopt a whole-systems approach to service provision.

5 Availability of multidisciplinary therapeutic care distinguishes good in-patient facilities from those that are no more than settings of containment. There should be regular monitoring of this availability through the care programme approach and other reviews.

6 All in-patient units should be able to show evidence of having gone through an external accreditation process such as the Royal College of Psychiatrists' peer review accreditation for forensic beds, AIMS-LD or any other equivalent.

7 All in-patient units should be able to show evidence of a minimum data-set of treatment outcomes that includes baseline descriptions and measures of efficacy, patient safety and patient experience.

(Adapted from Royal College of Psychiatrists 2013

in-patient psychiatric services will continue to be needed, particularly if continuous nursing observation or behavioural monitoring and professional input beyond what can be safely achieved in the community are required. Availability of this resource will therefore remain an important component of achieving equity of treatment outcomes for this often marginalised group of individuals.

\section{References}

Alexander RT, Piachaud J, Singh I (2001) Two districts, two models: inpatient care in the psychiatry of learning disability. British Journal of Developmental Disabilities, 47: 105-10.

Alexander RT, Piachaud J, Odebiyi L, et al (2002) Referrals to a forensic service in the psychiatry of learning disability. British Journal of Forensic Practice, 4: 29-33.
Alexander RT, Crouch K, Halstead S, et al (2006) Long-term outcome from a medium secure service for people with intellectual disability. Journal of Intellectual Disability Research, 50: 305-15.

Bjelogrlic-Laakso N, Aaltonen S, Dorn T, et al (2014) Need for special units for the management of neuropsychiatric disorders in people with intellectual disabilities. Acta Psychiatrica Scandinavica, 130: 77-9.

Care Quality Commission (2011) Count Me In 2010: Results of the 2010 National Census of Inpatients and Patients on Supervised Community Treatment in Mental Health and Learning Disability Services in England and Wales. Care Quality Commission.

Carroll A, Lyall M, Forrester A (2004) Clinical hopes and public fears in forensic mental health. Journal of Forensic Psychiatry \& Psychology, 15: 407-25.

Chaplin R (2004) General psychiatric services for adults with intellectual disability and mental illness. Journal of Intellectual Disability Research, 48: $1-10$.

Chaplin R (2009) New research into general psychiatric services for adults with intellectual disability and mental illness. Journal of Intellectual Disability Research, 53: 189-99.

Cooper S-A, Smiley E, Morrison J, et al (2007) Mental ill-health in adults with intellectual disabilities: prevalence and associated factors. British Journal of Psychiatry, 190: 27-35.

Cresswell J, Bleksley S, Lemmey S (eds) (2010) Accreditation for Inpatient Mental Health Services - Learning Disabilities (AIMS-LD): Standards for Adult Inpatient Learning Disability Units - Assessment and Treatment Units (2nd edn). Royal College of Psychiatrists Centre for Quality Improvement.

Department of Health (2001) Valuing People: A New Strategy for Learning Disability for the 21st Century (White Paper). Department of Health.

Department of Health (2012a) Department of Health Review: Winterbourne View Hospital: Interim Report. Department of Health.

Department of Health (2012b) Transforming Care: A National Response to Winterbourne View Hospital: Department of Health Review Final Report. Department of Health.

Department of Health (2015) Mental Health Act 1983: Code of Practice - 2015 Revision. TSO (The Stationery Office).

Emerson E, Baines S (2010) Health Inequalities \& People with Learning Disabilities in the UK: 2010. Learning Disability Observatory (http:// www.improvinghealthandlives.org.uk/uploads/doc/vid 7479 IHaL20103Healthlnequality2010.pdf).

Hall I, Parkes C, Higgins A, et al (2006a) The development of a new integrated mental health service for people with learning disabilities. British Journal of Learning Disabilities, 34: 82-7.

Hall I, Parkes C, Samuels S, et al (2006b) Working across boundaries: clinical outcomes for an integrated mental health service for people with intellectual disabilities. Journal of Intellectual Disability Research, 50: 598-607.

Health and Social Care Information Centre (2013) Learning Disabilities Census Report - England, 30th of September 2013. HSCIC

Holland T, Clare ICH, Mukhopadhyay T (2002) Prevalence of 'criminal offending' by men and women with intellectual disability and the characteristics of 'offenders': implications for research and service development. Journal of Intellectual Disability Research, 46: 6-20.

Hollins S (2000) Developmental psychiatry - insights from learning disability. British Journal of Psychiatry, 177: 201-6.

Kennedy HG (2002) Therapeutic uses of security: mapping forensic mental health services by stratifying risk. Advances in Psychiatric Treatment, 8: 433-43.

Lindsey M (2000) Services for people with learning disability and mental health problems. Mental Health Review, 5: 5-18.

Morgan VA, Leonard H, Bourke J, et al (2008) Intellectual disability cooccurring with schizophrenia and other psychiatric illness: populationbased study. British Journal of Psychiatry, 193: 364-72.

Moss S, Emerson E, Bouras N, et al (2002) Mental disorders and problematic behaviours in people with intellectual disability: future 
directions for research. Journal of Intellectual Disability Research, 41: $440-7$.

NHS Confederation (2012) Defining Mental Health Services: Promoting Effective Commissioning and Supporting OIPP. NHS Confederation.

NHS England (2013) Bed Availability and Occupancy Data - Overnight: Beds Time Series - 1987-88 to 2009-10. NHS England (http://www. england.nhs.uk/statistics/statistical-work-areas/bed-availability-andoccupancy/bed-data-overnight//.

Phillips N (ed) (2007) Standards for People with Learning Disabilities in Medium Secure Care. London.

Plant A, McDermott E, Chester V, et al (2011) Substance misuse among offenders in a forensic intellectual disability service. Journal of Learning Disabilities and Offending Behaviour, 2: 127-36.

Royal College of Psychiatrists, British Psychological Society, Royal College of Speech and Language Therapists (2007) Challenging Behaviour: A Unified Approach. Clinical and Service Guidelines for Supporting People with Learning Disabilities who are at Risk of Receiving Abusive or Restrictive Practices (College Report CR144). Royal College of Psychiatrists.

Royal College of Psychiatrists (2011) Future Role of Psychiatrists Working with People with Learning Disability (Faculty Report FR/LD/1). Faculty of the Psychiatry of Learning Disability, Royal College of Psychiatrists.

Royal College of Psychiatrists (2013) People with Learning Disability and Mental Health, Behavioural or Forensic Problems: The Role of In-Patient
Services (Faculty Report FR/ID/03). Faculty of Psychiatry of Intellectual Disability, Royal College of Psychiatrists.

Royal College of Psychiatrists (2014) Forensic Care Pathways for Adults with Intellectual Disability Involved with the Criminal Justice System (Faculty Report FR/ID/04). Faculty of Psychiatry of Intellectual Disability \& Faculty of Forensic Psychiatry, Royal College of Psychiatrists.

Tajuddin M, Nadkarni S, Biswas A, et al (2004) A study of the use of an acute inpatient unit for adults with learning disability and mental health problems in Leicestershire, UK. British Journal of Developmental Disabilities, 50: 59-68.

Tucker S, Hughes T (eds) (2007) Standards for Medium Secure Units: Quality Network for Medium Secure Units. Royal College of Psychiatrists' Centre for Quality Improvement

Tucker S, Iqbal M, Holder S (eds) (2012) Standards for Low Secure Services (CCOI publication number: CCOI 130). Royal College of Psychiatrists Centre for Quality Improvement.

World Health Organization (1992) The International Statistical Classification of Diseases and Related Health Problems, Tenth Revision (ICD-10). WHO

Xeniditis K, Russell A, Murphy D (2001) Management of people with challenging behaviour. Advances in Psychiatric Treatment, 7: 109-16.

\section{Cases}

$R v$ Hallstrom ex parte $W[1986] 2$ All ER 306.

\section{MCOs}

Select the single best option for each question stem

1 The rate of mental disorders in people with intellectual disability:

$\mathrm{a}$ is lower than that in people without an intellectual disability

$\mathrm{b}$ is the same as that in people without an intellectual disability

c is higher than that in people without an intellectual disability

$\mathrm{d}$ has not been measured before

$\mathrm{e}$ is lower in those in in-patient settings than those living in the community.

2 The proportion of adults with intellectual disability who are in contact with learning disability services in England is:

a $10 \%$

b $21 \%$

c $39 \%$ d $57 \%$

e $75 \%$.

3 The number of categories of in-patient bed described in the UK for people with intellectual disability and mental health problems is:

a 1

b 2

c 6

d 8

e 10 .

4 The bed category that constitutes secure in-patient care is:

a category 1

b category 2

c category 3

d category 4

e category 5
5 Which of the following is not a proposed minimum data-set measure of outcome variables?

a measure of effectiveness

b measure of patient safety

c number of in-patient beds

$\mathrm{d}$ measure of patient experience

e measure of aggression. 